\title{
Depth profiling of dopants implanted in Si using the synchrotron radiation based high- resolution grazing emission technique
}

\author{
Y. Kayser, ${ }^{a *}$ D. Banaś, ${ }^{b}$ W. Cao, ${ }^{a}$ J.-Cl. Dousse, ${ }^{a}$ J. Hoszowska, ${ }^{a}$ \\ P. Jagodziński, ${ }^{b}$ M. Kavčič, ${ }^{c}$ A. Kubala-Kukuś, ${ }^{b}$ S. Nowak, ${ }^{a}$ M. Pajek ${ }^{b}$ \\ and J. Szlachetko ${ }^{b, d}$
}

We report on the surface-sensitive grazing emission X-ray fluorescence technique combined with synchrotron radiation excitation and high-resolution detection to realize depth-profile measurements of Al-implanted Si wafers. The principles of grazing emission measurements as well as the benefits offered by synchrotron sources and wavelength-dispersive detection setups are presented. It is shown that the depth distribution of implanted ions can be extracted from the dependence of the $X$-ray fluorescence intensity on the grazing emission angle with nanometer-scale precision provided that an analytical function describing the shape of the depth distribution is assumed beforehand. If no $a$ priori assumption is made, except a bell shaped form for the dopant distribution, the profile derived from the measured angular distribution is found to reproduce quite satisfactorily the depth distribution of the implanted ions.

\section{Introduction}

The advances made over the last decade in the semiconductor industry are essentially because of the miniaturization of devices. Currently, the smallest commercially available devices are based on the $32 \mathrm{~nm}$ manufacturing process. Indeed, decreasing feature sizes offers the chance to further enhance the device speed and to design more and more complex integrated circuits leading, nowadays, to ultra large scale integrated circuits with more than a billion transistors. Moreover, the production cost per device unit and the power consumption decrease with the device size. However, in order to keep the aspect ratio of the devices constant, this down-scaling implies shorter channels and, therefore, increased leakage currents. This short-channel effect can be suppressed by designing devices based on ultra-shallow junctions, with junction depths of several tens of nanometers. For semiconductor doping based on ion implantation, this means that lower implantation energies need to be used. This, however, reduces the efficiency of the implantation process: because of space-charge effects the beam current is limited, resulting in longer production times. Also, the necessary consecutive thermal annealing to activate the dopants may cause the implanted ions to diffuse. Alternatives to ultra-low energy ion implantation combined with rapid thermal annealing are plasma ion immersion implantation ${ }^{[1]}$ and cluster ion implantation, ${ }^{[2]}$ which profits from the decreased charge density of the implanted molecules but produces more damage.

Independently of the implantation technique, the profiling of narrow junctions is quite challenging but necessary to survey the manufacturing processes and assist in further developments. The most common depth profiling technique, secondary ion mass spectroscopy (SIMS), struggles with the characterization of the depth and concentration distribution of dopants located within the first few nanometers below the surface. Despite recent progresses ${ }^{[3]}$ SIMS suffers from the formation of a transient region: until an equilibrium regime between the implanted and sputtered ion yields is established, SIMS delivers unreliable results. Therefore, SIMS, which is usually a very precise depth profiling technique, has difficulties to fully characterize narrow depth distributions located within the first tens of nanometers below the surface. Other depth profiling techniques like Auger electron spectroscopy or X-ray photoelectron spectroscopy are surface sensitive, the upper limit of $5-10 \mathrm{~nm}$ corresponding to the mean free path of the electrons, but have either difficulties or are unable to quantify the implanted species. The depth resolutions of high resolution Rutherford Backscattering and medium energy ion scattering have been improved, but both suffer from their low sensitivity towards low-Z elements.

Depth profiling methods based on X-ray fluorescence (XRF) like grazing emission X-ray fluorescence (GEXRF) have the great advantage to be non-destructive. Furthermore, these techniques

\footnotetext{
* Correspondence to: Y. Kayser, Department of Physics, University of Fribourg, $\mathrm{CH}-1700$ Fribourg, Switzerland.

E-mail: yves.kayser@unifr.ch

a Department of Physics, University of Fribourg, CH-1700 Fribourg, Switzerland

b Institute of Physics, Jan Kochanowski University, 25-406 Kielce, Poland

c J. Stefan Institute, SI-1000 Ljubljana, Slovenia
}

d European Synchrotron Radiation Facility (ESRF), F-38043 Grenoble, France 
are sensitive enough to detect trace amounts of impurities on the wafer surface or in the bulk. ${ }^{[4,5]}$ Depth profiling of implanted dopants by means of GEXRF is possible because of the refraction of the emitted fluorescence X-rays at the flat, polished sample surfaces. By observing the XRF intensity under different exit angles in the vicinity of the critical angle of total reflection, different depth regions are probed. The critical angle of total reflection is of the order of a few milliradian and depends on the density and the atomic number of the substrate, as well as the energy of the XRF line. Consequently, as it will be shown in this paper, the depth distribution of implanted ions can be reconstructed by measuring the intensity dependence of an X-ray emission line from the implanted species on the grazing exit angle defined relatively to the flat target surface.

Alternatively, an XRF based depth profiling approach can also be realized with grazing incidence XRF (GIXRF) ${ }^{[6]}$ or by combining GIXRF and SIMS approaches. ${ }^{[7]}$ GIXRF and GEXRF are physically equivalent in the sense that the refraction effect takes place on the same interface but from different incidence directions and for different X-ray energies (photon beam energy or fluorescence energy). Thus, similar results are to be expected from both techniques. In GIXRF, the XRF intensity is observed as a function of the incidence angle of the highly collimated monochromatic primary photon beam that is totally externally reflected for incidence angles below the critical angle. The different depth regions can be probed by varying the incidence angle. This modifies the X-ray standing wave field on top of the sample surface and the penetration range of the incident photons. Experimentally, GIXRF has a large solid angle of detection because the energy dispersive detector can be mounted close to the target surface. Consequently, GIXRF offers about one order of magnitude better detection limits for trace impurities compared with GEXRF. ${ }^{[8]}$ However, GIXRF setups are not versatile with respect to the type of the primary beam, and it is not possible to combine them with wavelength-dispersive detection setups to profit from the advantages related to the high energy resolution offered by the latter.

\section{GEXRF methodology}

The concept of grazing emission was first introduced in 1983. ${ }^{[9]}$ Different applications based on this technique have been realized since in cultural heritage ${ }_{,}^{[10]}$ detection of trace elements ${ }^{[11]}$ and contaminants on Si surfaces, ${ }^{[12]}$ thin-layer analysis, ${ }^{[13]}$ and analysis of surfaces. ${ }^{[14]}$ GEXRF permits a multi-elemental analysis and a specification of the deposition type, respectively contamination, in terms of quantity and structure (bulk, layers and films, residual particles). The operational principle of GEXRF consists in measuring the evolution of an excited XRF line around its critical angle of total reflection. As it can be seen in Fig. 1, only the first few nanometers in the depth direction contribute to the measured intensity for exit angles below the critical angle. For this angular range, $\mathrm{X}$-rays emitted by atoms located further away from the surface are considered as plane waves, which, when arriving at the sample-vacuum interface, are refracted according to the Fresnel laws of diffraction. Because the refractive index of solid samples is smaller than unity for X-rays, the detection setup is insensitive to X-rays emitted far from the sample surface. This results in a considerably enhanced surface sensitivity as the contribution of the bulk atoms to the fluorescence signal to the detected intensity is suppressed due to the refraction away from the flat, polished sample surface upon

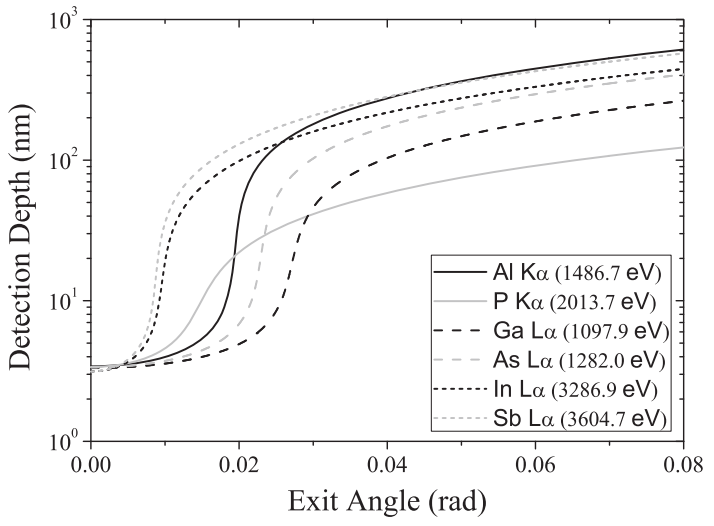

Figure 1. Evolution of the extinction length with the exit angle for selected $\mathrm{X}$-ray fluorescence lines of different n-type (gray) and p-type (black) dopants implanted in a Si wafer. The extinction length is the depth after which the intensity has dropped by a factor $\mathbf{e}^{-\mathbf{1}}$. It gives an estimate of the sample depth at which implanted dopants still contribute in a significant way to the production of the measured X-ray fluorescence signal.

the transition of the sample-vacuum interface. For exit angles larger than the critical angle of total reflection, the detection setup becomes sensitive to $\mathrm{X}$-rays emitted deeper inside the target (Fig. 1). In this angular range, the accessible depth region is limited by the self-absorption of the emitted X-rays: because of the grazing emission angle, the exit path of the emitted X-rays is quite large and varies with the sine of the exit angle. Different exit angles provide thus information from different depth regions allowing in principle to reconstruct the distribution of the emitting atoms.

For illustration, the variations of calculated angular profiles for different dopants implanted at $1 \mathrm{keV}$ into a Si wafer are shown in Fig. 2. Because the critical angle, corresponding to the steep increase of the detection depth curve (Fig. 1), varies as the inverse of the emission energy, the most pronounced features are concentrated at smaller exit angles and in narrower regions with increasing emission energies. A good angular resolution for GEXRF setups is therefore mandatory.

In GEXRF setups, the target is usually irradiated perpendicular to the surface. The primary beam can be either provided by an X-ray tube, ${ }^{[15]}$ an electron gun, ${ }^{[16]}$ a particle accelerator, ${ }^{[17]}$ or a synchrotron radiation (SR) source as reported in this paper. Indeed, one of

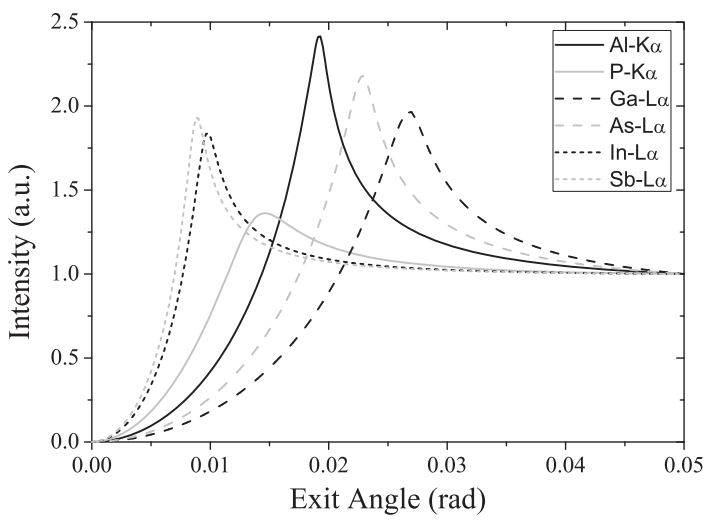

Figure 2. Calculated angular profiles for different typical dopants implanted at $1 \mathrm{keV}$ into $\mathrm{Si}$. The angular profiles depend on the dopant distribution and the energy of the emission line. For increasing emission energies, the angular profiles get narrower, which implies that for some elements, the $L \boldsymbol{\alpha}$ is preferred to the Ko line. The peak height depends on the absorption of the emitted X-rays in bulk Si. 
the advantages of GEXRF is its independence on the primary beam used to produce the fluorescence radiation. For depth profiling applications, photon based excitations are more convenient because of the larger penetration ranges. SR offers, besides the energy tunability, a high incident flux on the sample. Consequently, low detection limits can be achieved. ${ }^{[18]}$ Furthermore, SR sources can deliver focused and collimated X-ray beams, which can be easily combined with grazing emission setups to realize spatially $\mu$ m-resolved measurements. Elemental surface maps of the contaminant or dopant distribution in terms of location and concentration can thus be obtained. The feasibility of such elemental mapping was demonstrated in. ${ }^{[18]}$ At SR sources, GEXRF can be combined in addition with X-ray absorption spectroscopy that allows to study, for example, the local electronic structure of implanted dopants. ${ }^{[8,19]}$ SR enhances, thus significantly, the versatility of possible GEXRF applications.

Grazing emission measurements are point-by-point measurements, the change of the exit angle being usually realized by moving a light weighed energy-dispersive detector on a circle centered on the fluorescence spot on the sample surface (Fig. 3) or by rotating the sample. The advantage of the first option is a constant incidence angle of the primary beam. As for multilayer samples, oscillations due to interferences of multiply reflected $\mathrm{X}$-rays are observed in the angular profiles, ${ }^{[20]}$ the exit angles should be varied in steps of about $0.2 \mathrm{mrad}$. The angular resolution depends on a double slit collimator system and the detector distance from the fluorescence spot. Reported angular resolutions are of the order of milliradian. The surface sensitive characteristics of the grazing emission geometry counterbalance the small solid angles of detection, especially if combined with an intense primary beam as delivered by SR sources.

Because the solid angle of detection of grazing emission setups is small, the energy-dispersive detector can be replaced by a wavelength-dispersive detection setup, where a collimation of the fluorescence radiation is realized by the dispersive element. The advantage is a higher energy resolution, which results in a greater sensitivity to chemical states when measuring $X$-ray emission lines from a valence shell. A good separation of the many L-lines of mid$Z$ elements and/or M-lines of heavy elements can also be achieved. The background is usually considerably lower for wavelengthdispersive setups, resulting in an improved signal-to-background ratio and thus an increased sensitivity, especially for low-Z elements. The combination of the grazing emission geometry with a wavelength-dispersive detection setup is presented in the following section.

\section{Instrumentation and measurements}

The measurements of Al-implanted Si wafers were carried out with the high-resolution von Hamos crystal X-ray spectrometer of the University of Fribourg, ${ }^{[21]}$ which was installed at the European Synchrotron Radiation Facility (ESRF) ID21 beamline. The primary beam was produced by a wiggler and monochromatized by Ni/B $\mathrm{B}_{4} \mathrm{C}$ multilayers, and two Si mirrors tilted at an angle of $12 \mathrm{mrad}$ with respect to the incident radiation. The beam size was defined by a $2 \mathrm{~mm}$ pinhole, and the flux was about $2 \times 10^{11}$ photons per second. For the measurement of the $\mathrm{Si}-\mathrm{K} \alpha$ fluorescence line, beam energy of $2.000 \mathrm{keV}$ was chosen, whereas for the $\mathrm{Al}-\mathrm{K} \alpha$ fluorescence line, the selected beam energy was $1.582 \mathrm{keV}$. Because both energies were just above the $\mathrm{K}$ absorption edges of the considered elements, we could profit from the large photoelectric crosssections. In addition, for the measurements of the Al-K $\alpha$ line, the choice of the photon energy allowed to avoid the background contribution from the strong $\mathrm{Si}-\mathrm{K} \alpha$ fluorescence line and also, because of the high resolution of the spectrometer and the energy-tunability of the SR source, to separate the Al-K $\alpha$ fluorescence line from the Resonant Raman scattering of the Si L-shell. ${ }^{[22]}$ The excellent background conditions for different exit angles can be seen in Fig. 4. Note that the combination of beam energy tunability and high energy resolution detection is very helpful, if it comes to extracting the signal from a trace element of atomic number $Z$ located in (or on) a bulk material with atomic number $Z+1 .{ }^{[23]}$ In the case of $\mathrm{Al}$ on $\mathrm{Si}$, we achieved, in this way, a direct detection limit (i.e. without any pre-concentration technique) of $4 \times 10^{12}$ atoms per $\mathrm{cm}^{2} .{ }^{[24]}$ For other trace elements, similar direct detection limits were obtained. ${ }^{[18]}$

For the measurements of the $\mathrm{Si}$ and $\mathrm{Al}-\mathrm{K} \alpha$ lines, the von Hamos spectrometer was equipped with an ammonium dihydrogen phosphate ADP $(101)$ crystal $(2 d=10.642 \AA)$ cylindrically bent to a curvature radius of $25.4 \mathrm{~cm}$. The detector was a back-illuminated coupled charge device (CCD) camera $(1340 \times 400$ pixels of $20 \times 20 \mu m^{2}$ each, read-out speed $1 \mathrm{MHz}$ ) allowing for a positionsensitive detection. Originally, this spectrometer was not designed for realizing grazing emission experiments. However, the grazing emission conditions can be straightforwardly fulfilled. Indeed, only fluorescence X-rays that hit the crystal planes at the Bragg angle, defined by the energy of the $X$-rays and the crystal lattice spacing, are refracted towards the detector. Therefore, the diffracting crystal planes define with respect to the target surface, an emission direction for the detection of the selected emission line. Grazing emission conditions are thus achieved by turning the flat target
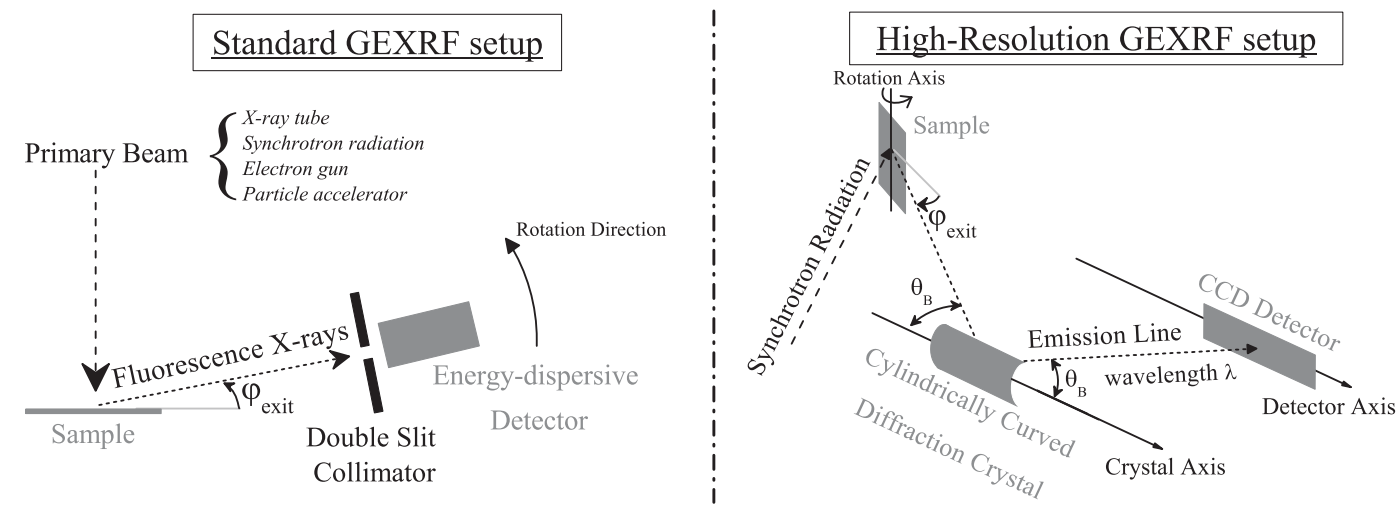

Figure 3. Comparison of a standard grazing emission X-ray fluorescence (GEXRF) setup with the presented SR-based high-resolution GEXRF setup. 


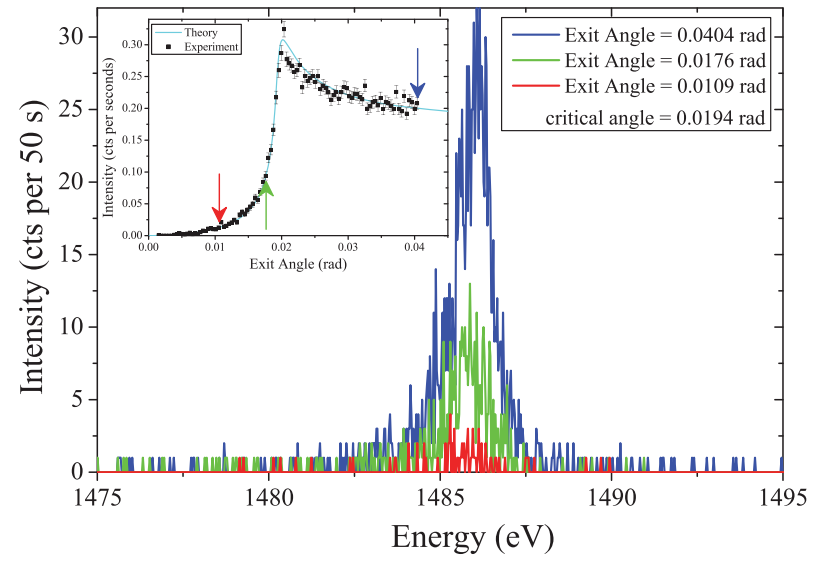

Figure 4. Experimental Al-K $\boldsymbol{\alpha}$ fluorescence X-ray spectra of the $10 \mathrm{keV}$ Al-implanted Si wafer measured at different exit angles, below, in the vicinity and above the critical angle (see arrows in the inset representing the corresponding angular profile). Extremely clean background conditions were obtained with our SR-based high-resolution setup.

surface close enough to this emission direction (Fig. 3). In this configuration, a fixed target position corresponds to a fixed exit angle and angular profiles are obtained by recording the intensity of the selected XRF line at different target positions. The samples' rotation axis is parallel to the sample surface and perpendicular to the incident beam direction. The crystal and detector positions, which depend on the fluorescence line to be measured and which define the solid angle of detection, are kept fixed while recording the fluorescence intensity for different exit angles. The exit angle can be varied with an excellent reproducibility by a stepping motor with a minimum step size of $40 \mu \mathrm{rad}$. The exit angle is only controlled on a relative scale and not on an absolute scale. A reference position is thus needed to associate to the different target positions, the corresponding exit angles. For the Alimplanted Si wafers, the calibration of the angular scale is realized with the critical angle of the $\mathrm{Si}-\mathrm{K} \alpha$ line, which is the same for bulk Si and implanted Si wafers, provided that the implantation fluence is low enough to not alter the optical properties of $\mathrm{Si}$. For the present measurements of the $\mathrm{Al}-\mathrm{K} \alpha$ and $\mathrm{Si}-\mathrm{K} \alpha$ lines, this condition is satisfied.

As in the von Hamos geometry, the CCD covers a given range of diffraction angles, and because the exit angle is defined with respect to the Bragg angle, for a fixed target position, the exit angle varies on the CCD along the dispersion direction. In order to obtain angular profiles with a sufficient angular resolution, regions of interest are defined on the CCD in both the horizontal and vertical directions. The vertical restriction is needed as the sample surface may not be perfectly perpendicular to the dispersion axis. Note that, in choosing the regions of interest, a compromise between intensity and angular resolution has to be made.

In order to reconstruct the depth distribution of the implanted Al ions, the Al-K $\alpha$ line was measured for a total of $100 \mathrm{~s}$ at 100 different exit angles with a step length of 0.4 mrad, whereas the $\mathrm{Si}-\mathrm{K} \alpha$ line was measured for $20 \mathrm{~s}$ at 40 different positions in steps of $1 \mathrm{mrad}$. These measurements were realized for nine different Al-implanted Si wafers, the implantation energies being 1, 5, 10, $15,20,25,30,50$ and $100 \mathrm{keV}$ and the implantation fluence $10^{16}$ atoms $/ \mathrm{cm}^{2}$. The latter three samples were prepared at the Institute of Electronic Materials Technology in Warsaw, Poland, the implantations at lower energies than $30 \mathrm{keV}$ were realized at the Ion Beam Physics and Materials Research Institute at the Forschungszentrum Dresden-Rossendorf in Germany.

\section{Results and discussion}

The depth profiling capabilities of the presented SR-based highresolution GEXRF method are discussed in the succeeding text for the case of the Al-implanted Si wafers. The experimental angular profiles were fitted using the equation for implanted samples from ${ }^{[20]}$ :

$$
I(\phi) \approx\left|t_{0}\right|^{2} \int_{z_{1}}^{z_{0}} N(z) \times e^{-2 \operatorname{lm}\left(k_{z}\right)\left(z_{0}-z\right)} \times|\mathrm{MR}|^{2} \mathrm{~d} z,
$$

where $\left|t_{0}\right|^{2}$ represents the change in the field strength upon the transmission of the emitted fluorescence $X$-rays across the samplevacuum interface, $z_{0}$ and $z_{1}$ the depth coordinates of the front and rear surfaces, $N(z)$ the dopant distribution (Fig. 5), and the exponential the absorption of X-rays in the sample. The refractive index $n$ depends on the wavelength $\lambda$ of the fluorescence radiation, $k_{z}=2 \pi / \lambda \times \sqrt{n^{2}-\cos ^{2} \phi}$, where $\phi$ is the exit angle, and MR stands for multiple reflections. The latter, however, can be neglected because there is only one sharp interface, the sample-vacuum interface, where the emitted X-rays are refracted. The aim of the fitting procedure was to determine $N(z)$, the depth distribution of the implanted ions. The Stopping and Range of lons in Matter (SRIM) ${ }^{[25]}$ calculations for Al implantations in Si suggest using a Gaussian to fit the angular profiles, the center and the width of the Gaussian being the free parameters. To elude the dependence on the starting values of the fit, each experimental angular profile was fitted several times with a least squares fitting method, and the mean of the returned values was taken as the final result for the considered sample. The standard deviation $\sigma$ of the returned values for both parameters allows to estimate the accuracy of the numeric values for the center and the width of each distribution. With this approach, very precise results in good agreement with SRIM calculations were obtained. ${ }^{[26]}$ In order to verify that the use of a Gaussian for $N(z)$ was the best choice, the angular profiles were also fitted with an asymmetric depth distribution, joined half Gaussians. The results of the fits of the angular profiles with both types of curves together with the accuracy estimation are shown in Table 1. The central positions of the peaks are for almost all the samples in an excellent agreement and have comparable accuracies, whereas the widths for the joined half Gaussians differ by about $1 \%$ or less and are very close to the widths of the simple Gaussians. Except for the samples implanted at 1 and $100 \mathrm{keV}$, the widths of the first half Gaussian are larger than the widths of the second one. The accuracy is however much better for the second half Gaussian than for the first one for which the values returned by the fitting procedure show

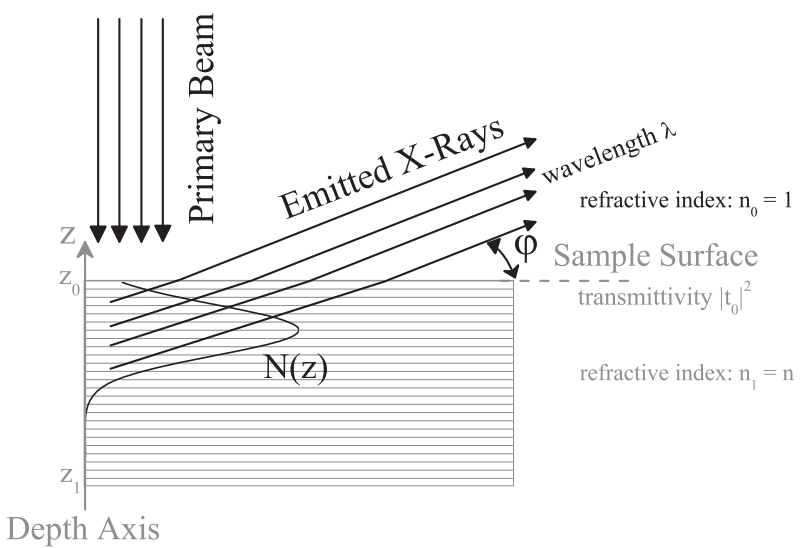

Figure 5. Definition of the parameters used in Eqn 1. 


\begin{tabular}{|lcccrr|}
\hline \multicolumn{5}{|l|}{ Table 1. Comparison of the experimentally extracted depth distributions using Gaussian and joined half-Gaussian functions } \\
\hline$E_{\text {Impl }}$ & $C_{\text {Gauss }}$ & $C_{\text {half-Gauss }}$ & $W_{\text {Gauss }}$ & $W_{1, \text { half-Gauss }}$ & $W_{2, \text { half-Gauss }}$ \\
\hline 1 & $4.01(0.49)$ & $4.44(0.73)$ & $1.92(0.03)$ & $1.9(0.7)$ & $1.96(0.10)$ \\
5 & $9.32(0.15)$ & $9.32(0.13)$ & $5.67(0.03)$ & $5.7(2.0)$ & $5.67(0.03)$ \\
10 & $18.3(0.2)$ & $18.3(0.2)$ & $9.53(0.03)$ & $9.9(3.2)$ & $9.53(0.03)$ \\
15 & $23.1(0.2)$ & $23.1(0.2)$ & $12.38(0.03)$ & $12.5(4.3)$ & $12.38(0.03)$ \\
20 & $32.7(0.4)$ & $32.7(0.3)$ & $16.51(0.03)$ & $17.0(6.1)$ & $19.8(6.9)$ \\
25 & $43.4(0.4)$ & $43.4(0.4)$ & $19.6(0.1)$ & $24.2(8.3)$ & $19.6(0.1)$ \\
30 & $56.8(0.6)$ & $56.8(0.4)$ & $24.1(0.1)$ & $36.1(12.3)$ & $24.1(0.1)$ \\
50 & $82.1(0.4)$ & $82.1(0.5)$ & $35.3(0.1)$ & $60.2(20.6)$ & $35.3(0.1)$ \\
100 & $169.0(0.7)$ & $169.0(0.5)$ & $61.0(0.2)$ & $61.0(0.1)$ \\
\hline
\end{tabular}

The implantation energy $E_{\mathrm{Impl}}$ of the $\mathrm{Al}$ ions is given in keV. $C$ stands for the center, $W$ for the width, both in units of nm. The index 1 for the width of the joined half-Gaussians represents the depth region extending from the surface to the center, the index 2 indicates the further depth region. The values in parentheses stand for the standard deviation $\sigma$ of the results retrieved by the described fitting procedures (see text).

a $\sigma$ of about $30 \%$. In all but one case, the widths of the second half Gaussian correspond to the widths of the simple Gaussian distributions and are retrieved with a similar accuracy. The surface concentrations obtained with joined half Gaussians are thus slightly larger, but the large relative error of the width of the first half Gaussian needs to be considered. The fits of the angular profiles are of the same quality for both types of distributions, showing that the initial choice of a symmetric distribution suggested by theory was well founded. Indeed, the angular profiles resulting from the fit with a Gaussian or joined half Gaussians are overlapping except in the region around the critical angle for the sample implanted at $1 \mathrm{keV}$, the sample where the absolute (and relative) difference between the central positions is the largest (Fig. 6). The depth profiles extracted from the fits of the angular intensity curves corresponding to the samples implanted at 1 and $20 \mathrm{keV}$ are depicted in Fig. 7. As shown, for both samples, a quite good agreement is observed between the experimental distributions and the theoretical ones obtained from SRIM calculations. ${ }^{[25]}$

In an alternative approach, the depth distributions of the implanted ions were extracted without any other a priori knowledge than the expected bell-shaped distribution. To this end, a triangular shape with regularly spaced points was first adopted to fit the experimental angular profiles. The aim was to determine the approximate depth region of the dopant distribution and its maximum concentration. A triangular shape seemed to be reasonable because, in general, implanted ions show (asymmetric) bellshaped distributions. A different polygonal shape can be assumed

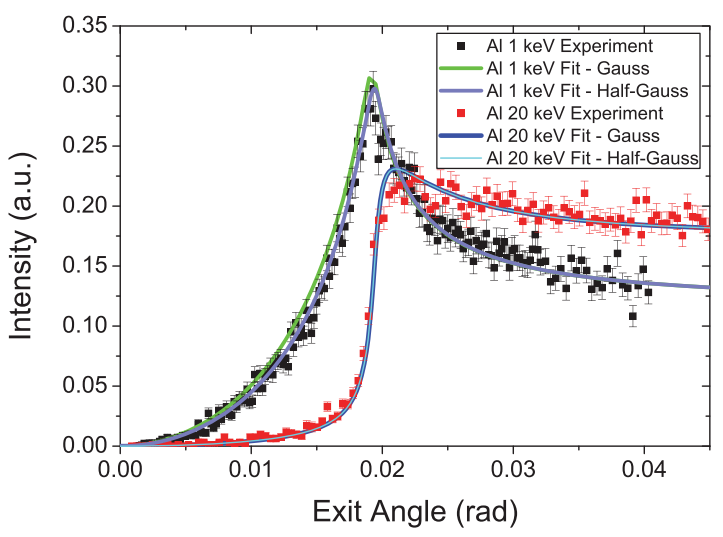

Figure 6. Comparison of the angular profiles returned by the fitting approach with a Gaussian and joined half Gaussians for the samples implanted at $1 \mathrm{keV}$ and $20 \mathrm{keV}$, respectively. if specific conditions, like a bimodal distribution, need to be satisfied. Actually, different bell-shaped polygonal shapes resulted in comparable final results, so that it can be assumed that the choice of the initial shape is not crucial. In the next step, only the depth coordinates $z_{i}(i=1, \ldots, p)$ of the $p$ points of the initial triangular distribution were allowed to vary within certain limits to improve the fit of the experimental angular profile, the concentration values $N\left(z_{i}\right)$ being kept fixed. The linear interpolation of the obtained set of point coordinates $\left(z_{i}, N\left(z_{i}\right)\right)$ was used as input to start an iteration process. In each iteration step, the depth resolution was improved by fitting the experimental angular profile with an increased number of points $p$ in the depth distribution curve returned by the preceding iteration step. The concentration values $N\left(z_{i}\right)$ of the initially regularly spaced points and subsequently the depth coordinates $z_{i}$ were allowed to vary within given limits defined by the starting values for the coordinates of each point and its immediate neighbors. This iteration process was stopped when the precision of the obtained point coordinates could not be improved further. As an example, the first result (triangle) of the fitting procedure is displayed in Fig. 8 together with the final result, the fit with joined half Gaussians and the SRIM predictions for the $25 \mathrm{keV}$ Al-implanted Si sample. The agreement with the theoretical depth distribution is quite good, the peak positions are quite close to each other, and the overall shapes are similar. The tails of the extracted depth distributions are, however, wider

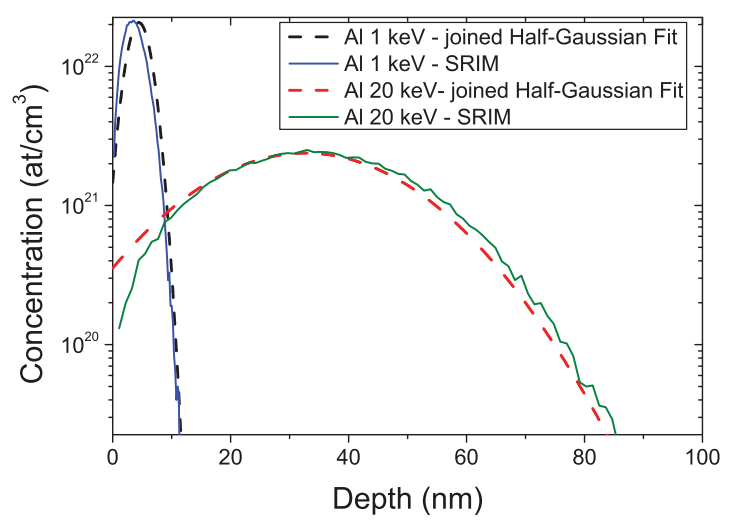

Figure 7. Experimental depth distributions extracted from the fits of the angular intensity curves shown in Fig. 6 (see also Table 1 for numerical values). The theoretical Stopping and Range of lons in Matter (SRIM) curves are shown for comparison. The curves have been scaled to the nominal implantation fluence of $10^{16} \mathrm{at} / \mathrm{cm}^{2}$. 


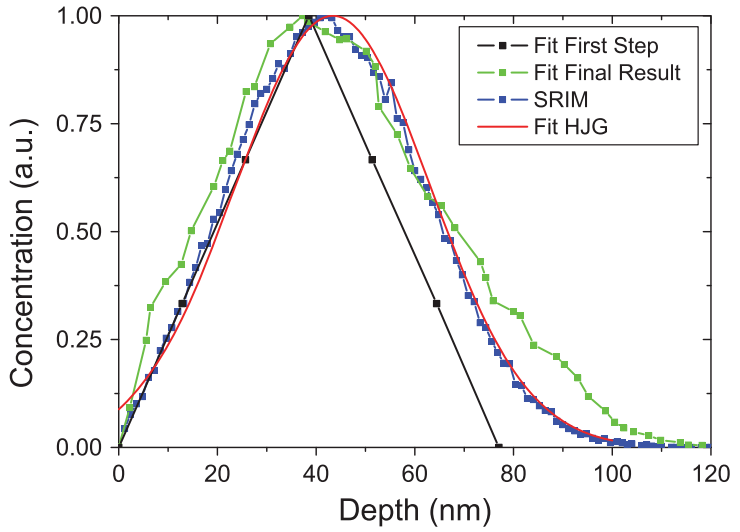

Figure 8. Illustration of the first step in the fitting procedure and the final result of the iteration procedure used to extract the depth distribution from the angular profile of the $25 \mathrm{keV}$ Al-implanted Si wafers. For comparison, the Stopping and Range of lons in Matter (SRIM) curve and the fit with joined half Gaussians are also represented. For details, see text.

than those of the theoretical one. This could be due to dopant diffusion following the implantation or it indicates an overestimation of the distribution tails by the fitting procedure. In the latter case. the determined junction depth, an important parameter for the microelectronic industry, would be too large. Nevertheless, because the shape of the depth distribution is not fixed beforehand, this approach may be promising to detect possible dopant diffusion in thermally annealed samples compared with asimplanted samples because the width or straggle of the distribution is not described by a single parameter. The angular profile corresponding to the final distribution obtained from the iteration procedure is depicted in Fig. 9. For comparison, the experimental data and the results of the SRIM calculations are also plotted. As shown, a quite good agreement is observed in both cases.

Concerning the quantification of the implanted dopants, the fluorescence intensity for exit angles far above the critical angle depends directly on the number of emitting atoms (Fig. 2). Equivalently, the area of the extracted depth distributions can be considered. The samples were found to be implanted within $\pm 10 \%$ with the same fluence. This error could be reduced by measuring at a well-defined exit angle, far above the critical angle, the XRF intensity with better statistics. However, for both approaches, in

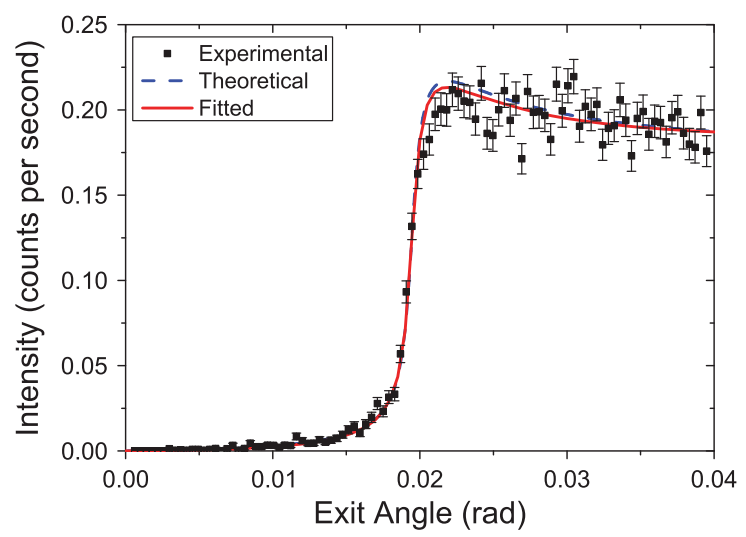

Figure 9. Angular profile corresponding to the final distribution of Fig. 8 (red solid line). The experimental data and the theoretical curve [Stopping and Range of lons in Matter (SRIM) + Eqn 1] for this sample are also shown for comparison. order to obtain absolute numbers, a reference sample with certified implantation fluence is needed.

\section{Conclusion}

In conclusion, the presented method profits from the advantages offered by synchrotron radiation, high energy resolution, and the grazing emission geometry to realize, despite the small solid angle of detection, depth-profiling measurements of Al-implanted Si wafers with a reasonable XRF yield excellent background conditions and a good angular resolution. From the measured angular profiles, it is possible to extract with great accuracy the depth distribution of implanted dopants by either assuming a given analytical shape derived from SRIM predictions for the distribution or by applying an iterative method that converges to the final result without any other a priori assumptions than a bell-shaped form for the dopant distribution curve. The possibility to detect dopant diffusion due to thermal annealing still needs to be assessed. For the as-implanted samples, a fair agreement with theory was observed for both presented approaches. Thus SR-based highresolution GEXRF method shows a great potential for characterizing narrow depth distributions.

\section{Acknowledgements}

The financial supports of the Swiss National Science Foundation and of the Polish Ministry of Science and Higher Education (special project ESRF/73/2006) and of the ESRF are acknowledged.

\section{References}

[1] P. K. Chu. Plasma Phys Control Fusion 2003, 45, 555.

[2] S. Heo, H. Hwang, H. T. Cho, W. A. Krull. Appl Phys Lett 2006, 89, 243516.

[3] W. Vandervorst. App/ Surf Sci 2008, 255, 805.

[4] R. Klockenkämper, M. Becker, H. Bubert, P. Burba, L. Palmetshofer. Anal Chem 1990, 62, 1674.

[5] Z. M. Spolnik, M. Claes, R. Van Grieken, P. K. de Bokx, H. P. Urbach. Spectrochim Acta Part B 1999, 54, 1525.

[6] P. Hönicke, B. Beckhoff, M. Kolbe, D. Giubertoni, J. van den Berg, G. Pepponi. Anal Bioanal Chem 2010, 396, 2825.

[7] G. Pepponi, D. Giubertoni, M. Bersani, F. Meirer, D. Ingerle, G. Steinhauser, C. Streli, P. Hönicke, B. Beckhoff. J Vac Sci Technol B 2010, 28, C1C59.

[8] F. Meirer, G. Pepponi, C. Streli, P. Wobrauschek, N. Zoeger. J Appl Phys 2009, 105, 074906.

[9] R. S. Becker, J. A. Golovchenko, J. R. Patel. Phys Rev Lett 1983, 50, 153.

[10] M. Claes, R. Van Ham, K. Janssens, R. Van Grieken, R. Klockenkämper, A. von Bohlen. Adv X-Ray Anal. 1998, 41, 262.

[11] M. Claes, K. van Dyck, H. Deelstra, R. van Grieken. Spectrochim Acta Part B 1999, 54, 1517.

[12] P. K. de Bokx, S. J. Kidd, G. Wiener, H. P. Urbach, S. De Gendt, P. Mertens, M. Heyns, Silicon Materials Science and Technology Proceedings, 98-1, The Electrochemical Society, Pennington, New Jersey, 1998, pp. 1511-1523.

[13] M. L. Monaghan, T. Nigam, M. Houssa, S. De Gendt, H. P. Urbach, P. K. de Bokx. Thin Solid Films 2000, 359, 197.

[14] T. Noma, K. Takada, A. lida. X-Ray Spectrom. 1999, 28, 433.

[15] P. K. de Bokx, C. Kok, A. Bailleul, G. Wiener, H. P. Urbach. Spectrochim Acta Part B 1997, 52, 829.

[16] K. Tsuji. Spectrochim Acta Part B 2005, 60, 1381.

[17] K. Tsuji, Z. M. Spolnik, K. Wagatsuma, R. Van Grieken, R. D. Vis. Anal Chem 1999, 71, 5033.

[18] J. Szlachetko, D. Banaś, A. Kubala-Kukuś, M. Pajek, W. Cao, J.-C. Dousse, J. Hoszowska, Y. Kayser, M. Szlachetko, M. Kavčič, M. Salome, J. Susini. J Appl Phys 2009, 105, 086101.

[19] M. A. Sahiner, P. Ansari, M. S. Carroll, C. A. King, Y. S. Suh, R. A. Levy, T. Buyuklimanli, M. Croft. Mat Res Soc Spring Meeting 2005, 864, E7.8.1-5(2005).

[20] H. P. Urbach, P. K. de Bokx. Phys Rev B 2001, 63, 085408. 
[21] J. Hoszowska, J.-C. Dousse, J. Kern, C. Rhême. Nucl Instrum Methods Phys Res A 1996, 376, 129.

[22] J. Szlachetko, J.-C. Dousse, J. Hoszowska, M. Pajek, R. Barrett, M. Berset, K. Fennane, A. Kubala-Kukuś, M. Szlachetko. Phys Rev Lett 2006, 97, 073001

[23] M. Kavčič, M. Zitnik, K. Bucar, J. Szlachetko. X-Ray Spectrom 2011, $40,2$.
[24] A. Kubala-Kukuś, D. Banaś, W. Cao, J.-C. Dousse, J. Hoszowska, Y. Kayser, M. Pajek, M. Salomé, J. Susini, J. Szlachetko, M. Szlachetko. Phys Rev B 2009, 80, 113305.

[25] J. F. Ziegler. SRIM-2003Nucl Instrum Meth B 2004, 219-220, 1027.

[26] Y. Kayser, D. Banaś, W. Cao, J.-C. Dousse, J. Hoszowska, P. Jagodziński, M. Kavčič, A. Kubala-Kukuś, S. Nowak, M. Pajek, J. Szlachetko. Spectrochim Acta Part B 2010, 65, 445 . 